\title{
Reversed S-Shaped Bifurcation Curve for a Neumann Problem
}

\author{
Hui Xing $\mathbb{D}^{1}{ }^{1}$ Hongbin Chen, ${ }^{2}$ and Ruofei Yao ${ }^{2}$ \\ ${ }^{1}$ Department of Mathematics, Xian Polytechnic University, Xi'an 710048, China \\ ${ }^{2}$ School of Mathematics and Statistics, Xian Jiaotong University, Xian 710049, China
}

Correspondence should be addressed to Hui Xing; xinghui210@163.com

Received 24 April 2018; Revised 21 June 2018; Accepted 4 July 2018; Published 1 August 2018

Academic Editor: Douglas R. Anderson

Copyright (C) 2018 Hui Xing et al. This is an open access article distributed under the Creative Commons Attribution License, which permits unrestricted use, distribution, and reproduction in any medium, provided the original work is properly cited.

\begin{abstract}
We study the bifurcation and the exact multiplicity of solutions for a class of Neumann boundary value problem with indefinite weight. We prove that all the solutions obtained form a smooth reversed S-shaped curve by topological degree theory, CrandallRabinowitz bifurcation theorem, and the uniform antimaximum principle in terms of eigenvalues. Moreover, we obtain that the equation has exactly either one, two, or three solutions depending on the real parameter. The stability is obtained by the eigenvalue comparison principle.
\end{abstract}

\section{Introduction}

The existence and multiplicity of solutions of Neumann problems have been investigated by many authors; see, for example, [1-5]. It is well known that determining the exact number of solutions of semilinear equations is usually a very difficult and challenging task. The results on exact multiplicity of solutions for Neumann problems are very few in the previous literature.

In this paper, we study the bifurcation and the exact multiplicity of solutions for the Neumann problem

$$
\begin{aligned}
u^{\prime \prime}+a(x) u-b(x) u^{3} & =\lambda f(x), \quad x \in(0,1), \\
u^{\prime}(0) & =u^{\prime}(1)=0,
\end{aligned}
$$

depending on a real parameter $\lambda$, where $a(x), b(x)$, and $f(x)$ are given continuous functions and $b(x)>0$ and $a(x)$ may change sign.

Llibre and Roberto [6] studied the existence and the stability of periodic solutions of Duffing equation

$$
x^{\prime \prime}+c x^{\prime}+a(t) x+b(t) x^{3}=\lambda h(t) .
$$

Chen and Li [7] also studied the Duffing equation (2) in a very particular case, i.e., $a(t) \equiv a>0, b(t)=1$, and $a, c$ constants. They obtained that (2) has exactly three T-periodic solutions. Lomtatidze et al. [8] also studied the existence of periodic solutions of Duffing type equations.
Tzeng et al. [9] also studied the global bifurcation and exact multiplicity of positive solutions of Dirichlet problem with cubic nonlinearity

$$
\begin{aligned}
u^{\prime \prime}+\lambda\left(-\varepsilon u^{3}+\sigma u^{2}-\kappa u+\rho\right) & =0, \quad-1<x<1, \\
u(-1) & =u(1)=0,
\end{aligned}
$$

using the time-map method, where $\varepsilon, \sigma, \kappa, \rho$ are constants. Equation (3) is an autonomous system, and the time-map method has been successfully employed to solve the problem (3), but it is not applicable to study the nonautonomous system (1). There are many results on exact multiplicity of solutions for the Dirichlet problems; see [9-12].

In [13], under Neumann boundary value conditions, the bifurcation of solutions to a logistic equation with harvesting has been investigated using the uniform antimaximum principle and Crandall-Rabinowitz bifurcation theorem. The uniform antimaximum principle plays an important role in proving the main results. More theories and applications of antimaximum principle can be seen, for example, $[14,15]$. As continuation of [13], in this paper, the bifurcation of solutions for a Neumann problem with cubic nonlinearity is investigated using the uniform antimaximum principle, Crandall-Rabinowitz bifurcation theorem, the topological degree theory, and the continuation method. More detailed results on bifurcation theory can be seen in $[16,17]$. The topological degree theory and the uniform antimaximum 
principle play an important role in proving the main results in this paper.

\section{Preliminaries}

Definition 1 (see [18]). We call a solution $u$ of the equation

$$
\begin{gathered}
u^{\prime \prime}+g(x, u)=f(x), \quad x \in(0,1), \\
u^{\prime}(0)=u^{\prime}(1)=0
\end{gathered}
$$

a stable solution if the principal eigenvalue $\mu_{1}\left(g_{u}(x, u)\right)$ of the equation

$$
\begin{gathered}
w^{\prime \prime}+g_{u}(x, u) w=-\mu w, \quad x \in(0,1), \\
w^{\prime}(0)=w^{\prime}(1)=0
\end{gathered}
$$

is strictly positive. The solution $u$ is unstable if the principal eigenvalue $\mu_{1}\left(g_{u}(x, u)\right)$ is negative.

Definition 2 (see [18]). We call a solution $u$ of the equation

$$
\begin{gathered}
u^{\prime \prime}+g(x, u)=f(x), \quad x \in(0,1), \\
u^{\prime}(0)=u^{\prime}(1)=0
\end{gathered}
$$

a nondegenerate solution if the linearized equation

$$
\begin{gathered}
w^{\prime \prime}+g_{u}(x, u) w=0, \quad x \in(0,1), \\
w^{\prime}(0)=w^{\prime}(1)=0
\end{gathered}
$$

does not admit any nontrivial solutions. The solution $u$ is degenerate (singular) if the linearized equation (7) has nontrivial solutions.

Definition 3 (see [19]). A mapping $G: X \longrightarrow Y(X$ and $Y$ are topological spaces) is said to be proper if for every compact set $K \subset Y$, the set $G^{-1}(K)$ is compact in $X$.

Lemma 4 (see [19]). Let $X$ be a real Banach space and $L$ a linear, compact map in $X$. Suppose that $\lambda \neq 0$ and $\lambda^{-1}$ is not an eigenvalue of L. Let $\Omega \subset X$ be an open bounded and $0 \in \Omega$. Then

$$
\operatorname{deg}(I-\lambda L, \Omega, 0)=(-1)^{m(\lambda)},
$$

where $m(\lambda)$ is the sum of the algebraic multiplicities of all the eigenvalues $\mu$ satisfying $\mu \lambda>1$, and $m(\lambda)=0$ if $L$ has no eigenvalues $\mu$ of this kind.

Lemma 5. Consider the eigenvalue problem

$$
\begin{gathered}
-u^{\prime \prime}(x)+u(x)=\mu u, \quad 0<x<1, \\
u^{\prime}(0)=u^{\prime}(1)=0 .
\end{gathered}
$$

Then (9) has the Green function

$$
G(x, s)= \begin{cases}\frac{\cosh (1-x) \cosh s}{\sinh 1}, & 0 \leq s \leq x \leq 1, \\ \frac{\cosh (1-s) \cosh x}{\sinh 1}, & 0 \leq x \leq s \leq 1,\end{cases}
$$

and

$$
\begin{aligned}
& \operatorname{deg}\left(I-\mu L, B_{R}, 0\right) \\
& \quad= \begin{cases}1, & \text { for }-\infty<\mu<1, \\
(-1)^{n+1}, & \text { for } n^{2} \pi^{2}+1<\mu<(n+1)^{2} \pi^{2}+1,\end{cases}
\end{aligned}
$$

where for any $R>0, B_{R}=\{u \in C[0,1]:\|u\| \leq R\}$ denotes $a$ ball of radius $R$, and $(L u)(x)=\int_{0}^{1} G(x, s) u(s) d s$.

Proof. It is easy to obtain (10) and the proof is omitted. The eigenvalue problem (9) is equivalent to the equation $u-\mu L u=$ 0 . The equation $u-\mu L u=0$ has nontrivial solutions if and only if $\mu^{-1}$ is the eigenvalue of the operator $L$. For $\mu>1$, the general solution of equation (9) is

$$
u(x)=c \cos (\sqrt{\mu-1} x)+d \sin (\sqrt{\mu-1} x) .
$$

By Neumann boundary value condition, we have

$$
-c \sqrt{\mu-1} \sin (\sqrt{\mu-1})=0
$$

When $c \neq 0$, we have $\mu=n^{2} \pi^{2}+1$, where $n \geq 0, n \in \mathbb{N}$. Therefore, $\lambda_{n}=\left(n^{2} \pi^{2}+1\right)^{-1}$ is the eigenvalue of $L$, where $n \geq 0, N\left(L-\lambda_{n}\right)$ is the one-dimensional subspace of Banach space $X$ spanned by $u_{n}=\cos (n \pi x)$.

For $n \geq 0$, Lemma 4 implies that

$$
\begin{aligned}
& \operatorname{deg}\left(I-\mu L, B_{R}, 0\right) \\
& \quad= \begin{cases}1, & \text { for }-\infty<\mu<1, \\
(-1)^{n+1}, & \text { for } n^{2} \pi^{2}+1<\mu<(n+1)^{2} \pi^{2}+1 .\end{cases}
\end{aligned}
$$

This completes the proof.

Consider the eigenvalue problem

$$
\begin{aligned}
u^{\prime \prime}+a(x) u+\mu u & =0, \quad x \in(0,1), \\
u^{\prime}(0) & =u^{\prime}(1)=0 .
\end{aligned}
$$

We recall some propositions listed as follows.

Proposition 6. It is well known that the problem (15) has the eigenvalues $\mu_{1}(a(x))<\mu_{2}(a(x)) \leq \mu_{3}(a(x)) \leq \cdots$. The first eigenvalue $\mu_{1}(a(x))$ is real and simple, and the corresponding eigenfunction does not change sign. When $a(x) \equiv 0$, we denote $\mu_{i}(0)$ by $\mu_{i}$. It is obvious that $\mu_{1}=0$ is the first eigenvalue and $\mu_{2}=\pi^{2}$ is the second eigenvalue of the eigenvalue equation

$$
\begin{gathered}
u^{\prime \prime}+\mu u=0, \quad x \in(0,1), \\
u^{\prime}(0)=u^{\prime}(1)=0 .
\end{gathered}
$$

Proposition 7. The comparison theorem of eigenvalues can be stated as follows: $\mu_{i}(a(x))$ is strictly decreasing in the sense that $a_{1}(x) \ll a_{2}(x)$ implies that $\mu_{i}\left(a_{1}(x)\right)>\mu_{i}\left(a_{2}(x)\right)$, where $a_{1}(x) \ll a_{2}(x)$, namely, $a_{1}(x) \leq a_{2}(x)$, with the strict inequality on a set of positive measure. 
Proposition 8. Suppose that $\mu_{k} \ll a(x) \ll \mu_{k+1}, k=1,2, \ldots$. Then the equation

$$
\begin{gathered}
u^{\prime \prime}+a(x) u=0, \quad x \in(0,1), \\
u^{\prime}(0)=u^{\prime}(1)=0
\end{gathered}
$$

does not admit any nontrivial solutions.

Proof. Argue by contradiction, (17) admits nontrivial solutions. Let $u(x)$ be a nontrivial solution of the equation (17). It follows from $\mu_{k} \ll a(x) \ll \mu_{k+1}$ that $\mu_{k}(a(x))<\mu_{k}\left(\mu_{k}\right)=0$ and $\mu_{k+1}(a(x))>\mu_{k+1}\left(\mu_{k+1}\right)=0$, for $k=1,2,3 \cdots$. It follows from (17) that 0 is the eigenvalue, contradiction. Therefore, (17) admits only trivial solution 0. Equation (17) does not admit any nontrivial solutions. This completes the proof.

In order to prove our main theorem using the topological degree theory, the following lemma is essential. In the following lemma, the weight function $a(x)$ may change sign.

Lemma 9. Suppose that $a(x) \ll \pi^{2}$ satisfies the equation

$$
\begin{aligned}
L_{a} u & =u^{\prime \prime}+a(x) u=0, \quad x \in(0,1), \\
u^{\prime}(0) & =u^{\prime}(1)=0 .
\end{aligned}
$$

Then

$$
\operatorname{deg}\left(I-A, B_{R}, 0\right)= \begin{cases}1, & \text { for } \mu_{1}(a(x))>0 \\ -1, & \text { for } \mu_{1}(a(x))<0\end{cases}
$$

where $(A u)(x)=\int_{0}^{1} G(x, s)(a(s)+1) u(s) d s, G(x, s)$ is defined in (10), for any $R>0, B_{R}=\{u \in C[0,1]:\|u\| \leq R\}$ denotes a ball of radius $R$.

Proof. Equation (18) is equivalent to the equation

$$
u(x)=\int_{0}^{1} G(x, s)(a(s)+1) u(s) d s .
$$

In (11), let $n=0$ and $\mu-1=\beta$; we have

$$
\begin{aligned}
\operatorname{deg} & \left(I-(\beta+1) L, B_{R}, 0\right) \\
\quad & \begin{cases}1, & \text { for }-\infty<\beta<0, \\
-1, & \text { for } 0<\beta<\pi^{2} .\end{cases}
\end{aligned}
$$

Next we calculate $\operatorname{deg}\left(I-A, B_{R}, 0\right)$. Let $H(t, u)=u-(1-t)(\beta+$ $1) L u-t A u$. Now we prove that $H(t, u) \neq 0$ for $\forall t \in[0,1]$ and $x \in \partial B_{R}$. Argue by contradiction and assume that $H(t, u)=0$; that is, for $\forall t \in[0,1]$, the equation

$$
\begin{aligned}
u^{\prime \prime}+(1-t) \beta u+\operatorname{ta}(x) u & =0, \quad x \in(0,1), \\
u^{\prime}(0) & =u^{\prime}(1)=0
\end{aligned}
$$

has solution $u$ satisfying $\|u\|=R$ on $\partial B_{R}$.
Case 1. When $a(x)$ does not change sign:

(i) For $a(x) \ll 0$, choose $\beta<0$; that is, for $\forall t \in[0,1]$, $((1-t) \beta+\operatorname{ta}(x))<0$, by Proposition 7 , we have $\mu_{1}((1-$ t) $\beta+\operatorname{ta}(x))>\mu_{1}(0)=0$. It follows that (22) has only trivial solution $u \equiv 0$. Contradiction, therefore, we have $H(t, u) \neq 0$ for $t \in[0,1], x \in \partial B_{R}$. Using the homotopy invariance we get

$$
\begin{aligned}
\operatorname{deg}\left(I-A, B_{R}, 0\right) & =\operatorname{deg}\left(H(1, \cdot), B_{R}, 0\right) \\
& =\operatorname{deg}\left(H(0, \cdot), B_{R}, 0\right) \\
& =\operatorname{deg}\left(I-(\beta+1) L, B_{R}, 0\right)=1 .
\end{aligned}
$$

Therefore, for $a(x) \ll 0$, that is, $\mu_{1}(a(x))>0$,

$$
\operatorname{deg}\left(I-A, B_{R}, 0\right)=1 .
$$

(ii) For $0 \ll a(x) \ll \pi^{2}$, choose $\beta \in\left(0, \pi^{2}\right)$, that is, for $\forall t \in[0,1], 0 \ll(1-t) \beta+t a(x) \ll \pi^{2}$, we have $\mu_{1}((1-t) \beta+$ $t a(x))<\mu_{1}(0)=0$ and $\mu_{2}((1-t) \beta+t a(x))>\mu_{2}\left(\pi^{2}\right)=0$. By Proposition 8, it follows that (22) has only trivial solution $u \equiv$ 0 , contradiction. Therefore, we have $H(t, u) \neq 0$ for $t \in[0,1]$, $x \in \partial B_{R}$. Using the homotopy invariance, we get

$$
\begin{aligned}
\operatorname{deg}\left(I-A, B_{R}, 0\right) & =\operatorname{deg}\left(H(1, \cdot), B_{R}, 0\right) \\
& =\operatorname{deg}\left(H(0, \cdot), B_{R}, 0\right) \\
& =\operatorname{deg}\left(I-(\beta+1) L, B_{R}, 0\right)=-1 .
\end{aligned}
$$

Therefore, for $\mu_{1}(a(x))<0, a(x) \ll \pi^{2}$, we have

$$
\operatorname{deg}\left(I-A, B_{R}, 0\right)=-1 .
$$

Case 2. When $a(x)$ changes sign, let $a(x)=a^{+}(x)-a^{-}(x)$, where $a^{+}(x)=\max \{a(x), 0\}, a^{-}(x)=\max \{-a(x), 0\}$.

(i) For $\mu_{1}(a(x))>0$, consider the following equation:

$$
\begin{aligned}
u^{\prime \prime}+\left(\operatorname{ta}^{+}(x)-a^{-}(x)\right) u & =0, \quad x \in(0,1), \\
u^{\prime}(0) & =u^{\prime}(1)=0 .
\end{aligned}
$$

where $t \in[0,1]$. Obviously,

$$
\begin{aligned}
& t a^{+}(x)-a^{-}(x) \leq a^{+}(x)-a^{-}(x)=a(x) \\
& \qquad t \in[0,1] .
\end{aligned}
$$

By Proposition 7 and (28), we have

$$
\mu_{1}\left(t a^{+}(x)-a^{-}(x)\right) \geq \mu_{1}(a(x))>0, \quad \forall t \in[0,1] .
$$

When $t=0$, the solution of (27) is

$$
u(x)=\int_{0}^{1} G(x, s)\left(-a^{-}(s)+1\right) u(s) d s .
$$

Let $\left(L_{1} u\right)(x)=\int_{0}^{1} G(x, s)\left(-a^{-}(s)+1\right) u(s) d s ;$ since $-a^{-}(s) \ll$ 0 , we have

$$
\operatorname{deg}\left(I-L_{1}, B_{R}, 0\right)=1
$$


The solution of (27) is

$$
u(x)=\int_{0}^{1} G(x, s)\left(t a^{+}(s)-a^{-}(s)+1\right) u(s) d s .
$$

Let $h_{1}(t, u)=u-t A u-(1-t) L_{1} u$. It is easy to prove that $h_{1}(t, u) \neq 0$ for $\forall t \in[0,1], u \in \partial B_{R}$. Therefore, using the homotopy invariance, we get

$$
\begin{aligned}
\operatorname{deg}\left(I-A, B_{R}, 0\right) & =\operatorname{deg}\left(h_{1}(1, \cdot), B_{R}, 0\right) \\
& =\operatorname{deg}\left(h_{1}(0, \cdot), B_{R}, 0\right) \\
& =\operatorname{deg}\left(I-L_{1}, B_{R}, 0\right)=1 .
\end{aligned}
$$

(ii) For $\mu_{1}(a(x))<0, a(x) \ll \pi^{2}$, by Proposition 7 , $\mu_{2}(a(x))>0$; similarly, let

$$
\left(L_{2} u\right)(x)=\int_{0}^{1} G(x, s)\left(a^{+}(s)+1\right) u(s) d s .
$$

Since $a^{+}(s) \gg 0$, we have

$$
\operatorname{deg}\left(I-L_{2}, B_{R}, 0\right)=-1 .
$$

Let $h_{2}(t, u)=u-t A u-(1-t) L_{2} u$. It is easy to prove that $h_{2}(t, u) \neq 0$ for $\forall t \in[0,1], u \in \partial B_{R}$. Therefore, using the homotopy invariance we get

$$
\begin{aligned}
\operatorname{deg}\left(I-A, B_{R}, 0\right) & =\operatorname{deg}\left(h_{2}(1, \cdot), B_{R}, 0\right) \\
& =\operatorname{deg}\left(h_{2}(0, \cdot), B_{R}, 0\right) \\
& =\operatorname{deg}\left(I-L_{2}, B_{R}, 0\right)=-1 .
\end{aligned}
$$

This completes the proof.

Definition 10 (see [20]). Let $\Omega$ be open set in Banach space $E$. Suppose $A: \bar{\Omega} \longrightarrow E$ is completely continuous operator, $f=I-A$. Let $x_{0} \in \Omega$ be an isolated fixed point of $f$ in $\Omega$; that is, there exists $r>0$, such that $B_{r}=\left\{x:\left\|x-x_{0}\right\|<r\right\} \subset \Omega$, and $x_{0}$ is only fixed point of $f$ in $\bar{B}_{r}$. Define

$$
\text { ind }\left(I-A, x_{0}\right)=\operatorname{deg}\left(I-A, B_{r}, 0\right) \text {. }
$$

Lemma 11 (see [20]). Let $\Omega$ be open set in Banach space E. Suppose $A: \bar{\Omega} \longrightarrow E$ is completely continuous operator; $A$ has no fixed point on $\partial \Omega$. Suppose that there are finite isolated fixed points $x_{1}, x_{2}, \ldots, x_{m}$. Then

$$
\operatorname{deg}(I-A, \Omega, 0)=\sum_{i=1}^{m} \operatorname{ind}\left(I-A, x_{i}\right) .
$$

Lemma 12 (see [20]). Let $\Omega$ be open set in Banach space E. Suppose $A: \bar{\Omega} \longrightarrow E$ is completely continuous operator, $x_{0} \in$ $\Omega, A x_{0}=x_{0}$. Suppose that $A$ is Fréchet differentiable at $x_{0}$ and 1 is not the eigenvalue of derived operator $A^{\prime}\left(x_{0}\right)$. Then $x_{0}$ is an isolated fixed point of $A$ and

$$
\operatorname{ind}\left(I-A, x_{0}\right)=\operatorname{ind}\left(I-A^{\prime}\left(x_{0}\right), 0\right)=(-1)^{\beta} \text {, }
$$

where $\beta$ is the sum of the algebraic multiplicities of all the eigenvalues $A^{\prime}\left(x_{0}\right)$ in $(0,1)$.
Now we state three known results, which plays a key role in proving the main results in this paper. We do not provide their proof which can be seen in [21].

Lemma 13. Suppose that $a(x), a_{1}(x)$, and $a_{2}(x) \in C[0,1]$ such that

$$
a(x), a_{1}(x), a_{2}(x) \ll \pi^{2} .
$$

Then

(1) the possible solution $u$ of (18) is either $u(x) \equiv 0$ or $u(x) \neq 0$ for each $x \in[0,1]$;

(2) $L_{a} u=0(i=1,2)$ cannot both admit nontrivial solutions if $a_{1}(x) \ll a_{2}(x)$.

Lemma 14. Let $f(x)>0$ on $[0,1]$ and $a(x)$ satisfy

$$
a(x) \ll \frac{\pi^{2}}{4} .
$$

If $u(x)$ is a solution of the nonhomogeneous differential equation

$$
\begin{aligned}
u^{\prime \prime}+a(x) u & =f(x), \quad x \in(0,1), \\
u^{\prime}(0) & =u^{\prime}(1)=0,
\end{aligned}
$$

then the following statements hold:

(1) either $u(x)>0$ or $u(x)<0$ for all $x \in[0,1]$;

(2) maximum principle: $u(x)<0$, if $\mu_{1}(a(x))>0$;

(3) uniform antimaximum principle: $u(x)>0$, if $\mu_{1}(a(x))<0$.

For the reader's convenience, we denote the continuous function $g(x, u)=a(x) u-b(x) u^{3}$ in (1). The assumptions on function $g(x, u)$ are listed as follows:

(f1) $g(x, u)$ is locally differentiable with respect to the second variable with $g_{u}(x, u) \ll \pi^{2}$;

(f2) $\liminf _{u \longrightarrow \pm \infty} u(g(x, u)-\lambda f(x))<0$, uniformly in $x \in$ $[0,1]$.

Lemma 15. Let $X$ be an order Banach space and $[w, v]=\{u \in$ $X \mid w \leq u \leq v\}$ an order set. Assume that the given continuous function $g(x, u)$ satisfies $(f 1)$. Then

(1) the solutions of (1) are totally ordered;

(2) (1) cannot admit three distinct solutions in $[w, v]$ if $g_{u}(x, u)$ is strictly increasing or strictly decreasing in $[w, v]$.

For the convenience, we recast (1) in the operator form

$$
\begin{aligned}
F(\lambda, u):=u^{\prime \prime}+g(x, u)- & \lambda f(x), \\
& \mathbb{R} \times C^{2}[0,1] \longrightarrow C[0,1] .
\end{aligned}
$$


Lemma 16 (see [16]). Let $X$ and $Z$ be Banach spaces. Assume that $F: \mathbb{R} \times U \longrightarrow Z$ is continuously differentiable on $V \times U \subset$ $\mathbb{R} \times X$ satisfying the following three conditions:

(1) $F\left(\lambda_{*}, u_{*}\right)=0$ for some $\left(\lambda_{*}, u_{*}\right) \in V \times$ $U, \operatorname{dim} N\left(D_{u} F\left(\lambda_{*}, u_{*}\right)\right)=1$ and the null space $N\left(D_{u} F\left(\lambda_{*}, u_{*}\right)\right)=\operatorname{span}\{w\}$

(2) the Fredholm index of $D_{u} F\left(\lambda_{*}, u_{*}\right)$ is zero, $\operatorname{codim} R\left(D_{u} F\left(\lambda_{*}, u_{*}\right)\right)=1$;

(3) $D_{\lambda} F\left(\lambda_{*}, u_{*}\right) \notin R\left(D_{u} F\left(\lambda_{*}, u_{*}\right)\right)$.

Then there is a continuously differentiable curve through $\left(\lambda_{*}, u_{*}\right)$; that is, there exists $\left\{(\lambda(s), u(s))=\left(\lambda_{*}+\tau(s), u_{*}+\right.\right.$ $\left.s w+z(s)) \mid s \in(-\delta, \delta),(\lambda(0), u(0))=\left(\lambda_{*}, u_{*}\right)\right\}$ such that

$$
F(\lambda(s), u(s))=0, \quad \text { for } s \in(-\delta, \delta),
$$

and all solutions of $F(\lambda, u)=0$ in a neighborhood of $\left(\lambda_{*}, u_{*}\right)$ belong to the curve, $\tau(0)=\tau^{\prime}(0)=z(0)=z^{\prime}(0)=0$.

\section{Main Results}

Lemma 17. Assume ( $f 2$ ) holds. Then for every fixed $\lambda$, (1) has at least a solution and

$$
\operatorname{deg}\left(I-A, B_{R}, 0\right)=1
$$

for $R$ large enough, where $(A u)(x)=\int_{0}^{1} G(x, s)(g(s, u(s))+$ $u(s)-\lambda f(s)) d s$ and $G$ is defined in (10).

Proof. It follows from (f2) that there exists $R>0$ large enough such that $g(x, R)-\lambda f(x)<0$ and $g(x,-R)-\lambda f(x)>0$. Therefore, $-R$ and $R$ are subsolution and supersolution of (1), respectively. Therefore, there exists at least one solution of (1) between $-R$ and $R$. Next, we show that all the solutions of (1) are between $-R$ and $R$ for $R>0$ large enough. Argue by contradiction, suppose that there exists $x_{0}$ such that $u(x)$ attains its maximum value and $u\left(x_{0}\right)>R$. We have

$$
\begin{aligned}
& u^{\prime \prime}\left(x_{0}\right)+a\left(x_{0}\right) u\left(x_{0}\right)-b\left(x_{0}\right) u^{3}\left(x_{0}\right)-\lambda f\left(x_{0}\right) \\
& \quad=0
\end{aligned}
$$

It follows from $u^{\prime}\left(x_{0}\right)=0$ and $u^{\prime \prime}\left(x_{0}\right)<0$ that

$$
a\left(x_{0}\right) u\left(x_{0}\right)-b\left(x_{0}\right) u^{3}\left(x_{0}\right)-\lambda f\left(x_{0}\right) \geq 0 .
$$

From the above there exists $R>0$ large enough such that $g(x, R)-\lambda f(x)<0$, contradiction. Similar, suppose that there exists $x_{1}$ such that $u(x)$ attains its minimum value and $u\left(x_{1}\right)<-R$; we will obtain contradiction. Therefore, there exists $R>0$ large enough such that $\|u\|<R$ for all the solutions $u(x)$. (1) is equivalent to the following equation:

$$
u(x)=(A u)(x) .
$$

Next we calculate $\operatorname{deg}\left(I-A, B_{R}, 0\right)$. Let $h(t, u)=u-t A u, \forall t \in$ $[0,1]$. The equation $h(t, u)=0$ is equivalent to the following equation:

$$
u^{\prime \prime}+\operatorname{tg}(x, u)-(1-t) u-t \lambda f(x)=0
$$

It is evident that $-R$ and $R$ are subsolution and supersolution of (49), respectively. Therefore, all the solutions of (49) must be between $-R$ and $R$; that is, the equation $h(t, u)=0$ has no solution on $\partial B_{R}$. Therefore, $h(t, u) \neq 0$ for $t \in[0,1], u \in \partial B_{R}$. By the homotopy invariance properties of the topological degree we have

$$
\begin{aligned}
\operatorname{deg}\left(I-A, B_{R}, 0\right) & =\operatorname{deg}\left(h(\cdot, 1), B_{R}, 0\right) \\
& =\operatorname{deg}\left(h(\cdot, 0), B_{R}, 0\right) \\
& =\operatorname{deg}\left(I, B_{R}, 0\right)=1 .
\end{aligned}
$$

This completes the proof.

Lemma 18. Suppose that $\mu_{1}(a(x))<0, a(x) \ll \pi^{2}$, and $f(x)>0$ for all $x \in[0,1]$. Then (1) has exactly three solutions $u_{0}(x), 0, v_{0}(x)$ for $\lambda=0$, where $u_{0}(x)$ is the unique positive stable solution and $v_{0}(x)$ is the unique negative stable solution.

Proof. For the mapping $F$ defined in (43), let $\lambda=0$, and we have

$$
F(u)=u^{\prime \prime}+a(x) u-b(x) u^{3} .
$$

Next we prove that any solution $u(x)$ of $F(u)=0$ is nondegenerate.

First, we have the Fréchet derivative of (51)

$$
F_{u}(u)[w]=w^{\prime \prime}+\left(a(x)-3 b(x) u^{2}\right) w .
$$

It is obvious that $u=0$ is the solution of $F(u)=0$, and therefore, when $u=0, a(x)-3 b(x) u^{2}=a(x)$ in (52). It follows from $\mu_{1}(a(x))<0$ that 0 is nondegenerate.

Next, we prove that any nontrivial solution $u(x)$ of $F(u)=$ 0 is nondegenerate. $F(u)=0$ is equivalent to the following equation:

$$
u(x)=(A u)(x)
$$

where $(A u)(x)=\int_{0}^{1} G(x, s)(g(s, u(s))+u(s)) d s$ and $G$ is defined in (10). The equation

$$
w-A^{\prime}(u) w=0
$$

is equivalent to $F_{u}(u)[w]=0$, where $A^{\prime}(u)$ denotes the derivative operator of $A$. When $\mu_{1}(a(x))<0, a(x) \ll \pi^{2}$, by Lemma 9, Definition 10, and Lemma 12, there exists $R>0$, and we have

$$
\begin{aligned}
\text { ind }(I-A, 0) & =\operatorname{ind}\left(I-A^{\prime}(0), 0\right) \\
& =\operatorname{deg}\left(I-A, B_{R}, 0\right)=-1 .
\end{aligned}
$$

If $u(x)$ is a nontrivial solution of $F(u)=0$, it follows from Lemma 13 that $u(x) \neq 0$ for all $x \in[0,1]$. Therefore,

$$
\begin{aligned}
g_{u}(x, u) & =a(x)-3 b(x) u^{2}(x)<\frac{g(x, u)}{u} \\
& =a(x)-b(x) u^{2}(x) \ll \pi^{2} .
\end{aligned}
$$


Let $q_{1}(x)=g(x, u) / u$ and $q_{2}(x)=g_{u}(x, u)$. It follows from the above hypothesis that $u(x)$ is a nontrivial solution of $L_{q_{1}} u=0$. By the second conclusion of Lemma 13, we have that $L_{q_{2}} u=0$ does not have nontrivial solution, which implies that $u(x)$ is a nondegenerate solution.

Finally, we prove the positive solution is unique and stable. We denote by $u_{0}$ the nontrivial solution of (51). It is obvious that $-u_{0}(x)$ is also the nontrivial solution of (51). It follows from (56) and the comparison of eigenvalues that $\mu_{1}\left(q_{2}(x)\right)>\mu_{1}\left(q_{1}(x)\right)=0$. By Lemma 9, Definition 10, and Lemma 12, we have

$$
\text { ind } \begin{aligned}
(I-A, u) & =\operatorname{ind}\left(I-A^{\prime}(u), 0\right) \\
& =\operatorname{deg}\left(I-A, B_{R}, 0\right)=1 .
\end{aligned}
$$

Let $m$ be the number of nontrivial solutions of $F(u)=0$. Hence, $F$ is proper. Since 0 is a regular value of $F, m$ must be finite. According to Lemma 17, we have $\operatorname{deg}\left(I-A, B_{R}, 0\right)=1$ for sufficiently large $R$. By Lemmas 9 and 11 and the index formula, we have that

$$
\begin{aligned}
1 & =\operatorname{deg}\left(I-A, B_{R}, 0\right) \\
& =\operatorname{ind}(I-A, 0)+\sum_{u \neq 0} \operatorname{ind}\left(I-A, u_{i}\right)=-1+m .
\end{aligned}
$$

It is obvious that $m=2$. Let $v_{0}(x) \equiv-u_{0}(x)$ for $x \in[0,1]$. Therefore, $F(u)=0$ has exactly three solutions $u_{0}, 0$, and $v_{0}$. Since $\mu_{1}\left(q_{2}\right)>\mu_{1}\left(q_{1}\right)=0$, we have that the positive solution $u_{0}(x)$ is unique and stable. The negative solution $v_{0}(x)$ is also stable. This completes the proof.

Lemma 19. Suppose that $a(x) \ll \pi^{2} / 4$. For some $\lambda<0$, (1) has a unique positive solution $u_{1}(x)$ with $u_{1}(x)>u_{0}(x)$, where $u_{0}(x)$ is the unique positive solution of (1) for $\lambda=0$.

Proof. By Lemma 18, for $\lambda=0$ (1) has the unique positive solution $u_{0}$, which provided a subsolution of (1) for $\lambda<0$. There exists $R>0$ large enough such that $g(x, R)-\lambda f(x)<$ 0 . Thus, $R$ is a supersolution of (1). Therefore, we prove that there exists a positive solution $u_{1}(x)$ of (1) for $\lambda<0$ such that $u_{0}(x)<u_{1}(x)<R$.

Next we will prove that the positive solution $u_{1}$ is unique. Assume, by contradiction that (1) has another positive solution $u(x)$ for $\lambda<0$. Let $v(x)=u(x)-u_{1}(x) \neq 0$; then $w$ satisfies the following equation:

$$
\begin{aligned}
v^{\prime \prime} & +a(x) v-b(x)\left[u^{2}(x)+u(x) u_{1}(x)+u_{1}^{2}(x)\right] v \\
& =0 .
\end{aligned}
$$

Clearly, $a(x)-b(x)\left[u^{2}(x)+u(x) u_{1}(x)+u_{1}^{2}(x)\right]<a(x) \ll$ $\pi^{2} / 4$. By Lemma 13, $v(x) \equiv 0$ is the solution of (59), which is a contradiction. Therefore, the solution $u_{1}$ obtained above is only positive solution. For $\lambda<0, u_{1}$ satisfies (1) and

$$
u_{1}^{\prime \prime}+q(x) u_{1}=\lambda f(x)
$$

where $q(x)=a(x)-b(x) u_{1}^{2}(x)$. Again since $f(x)>0$ and $\lambda<0$, it follows from Lemma 14 that $\mu_{1}(q(x))>0$. Consider the linearization associated with (1)

$$
w^{\prime \prime}+\left(a(x)-3 b(x) u^{2}(x)\right) w=0
$$

where $g_{u}(x, u)=a(x)-3 b(x) u^{2}(x)<q(x)$. Thus, $\mu_{1}\left(g_{u}(x, u)\right)>\mu_{1}(q(x))>0$. Therefore, by Lemma 13 we have $u_{1}(x)$ is nondegenerate. This completes the proof.

Lemma 20. For $\lambda>0$, (1) has a unique negative solution $v_{1}(x)$ with $v_{1}(x)<v_{0}(x)$, where $v_{0}(x)$ is the unique negative solution of (1) for $\lambda=0$.

Proof. The proof is similar to the proof of Lemma 19.

Theorem 21. Assume that the first eigenvalue $\mu_{1}(a(x))<0$ and $f(x)>0$ for all $x \in[0,1]$. Suppose that $a(x) \ll$ $\pi^{2} / 4$. Then all the solutions of (1) are of one sign and lie on a unique reversed $S$-shaped solution curve, which is symmetric with respect to the origin. More precisely, there exists $\lambda_{*}>0$, such that

(i) For $\lambda>\lambda_{*}$, (1) has no positive solution and has a unique negative solution which is stable.

(ii) For $\lambda= \pm \lambda_{*}$, (1) has exactly two solutions. Moreover, when $\lambda=\lambda_{*}$, the negative solution is stable and the positive solution is degenerate. When $\lambda=-\lambda_{*}$, the positive solution is stable and the negative solution is degenerate.

(iii) For $-\lambda_{*}<\lambda<\lambda_{*}$, (1) has exactly three ordered solutions at the same $\lambda$ and the middle solution is unstable and the remaining two are stable. Moreover, when $\lambda<0$, the maximal solution is positive and the other two are negative. When $\lambda>0$, the minimal solution is negative and the other two are positive.

(iv) For $\lambda<-\lambda_{*}$, (1) has no negative solution and has a unique positive solution which is stable.

Proof. It follows from Lemma 19 that (1) has a unique nondegenerate positive solution $u_{1}(x)$ for some $\lambda<0$. The solution curve can be continued a little bit such that $u_{1}(x)$ remains positive for increasing $\lambda$ when $\lambda<0$. By Lemma 18 , (1) has a unique nondegenerate positive solution $u_{0}(x)$ for $\lambda=0$. The positive solution curve can pass through $u_{1}(x)$ and $u_{0}(x)$ and can be continued further for increasing $\lambda$ until the linearized equation (61) admits the nontrivial solutions. We claim that the curve of positive solutions cannot be continued for $\lambda>\lambda_{*}$. Next we will prove the existence of $\lambda_{*}$. Since $\mu_{1}(a(x))<0$, let $v(x)>0$ be the first eigenfunction of the problem

$$
\begin{gathered}
v^{\prime \prime}+a(x) v+\mu v=0, \quad x \in(0,1), \\
v^{\prime}(0)=v^{\prime}(1)=0 .
\end{gathered}
$$


Multiplying (1) by $v(x)$ and subtracting from (62) multiplied by $u$, after that integrating over $[0,1]$, we obtain

$$
\begin{gathered}
\int_{0}^{1}\left[b(x) u^{3}(x)+\mu u(x)\right] v(x) d x \\
\quad=-\lambda \int_{0}^{1} f(x) v(x) d x .
\end{gathered}
$$

Applying the mean-value theorem for (63), there exists $\xi \in$ $[0,1]$ such that

$$
\begin{aligned}
h(u(\xi), b(\xi)) & =b(\xi) u^{3}(\xi)+\mu_{1} u(\xi) \\
& =-\frac{\lambda \int_{0}^{1} f(x) v(x) d x}{\int_{0}^{1} v(x) d x} .
\end{aligned}
$$

For all $b(\xi) \leq b_{0}=\max _{x \in[0,1]} b(x)$, the only root of $h(u(\xi), b(\xi))=m$ is negative for $m<h\left(\sqrt{-\mu_{1} / 3 b_{0}}, b_{0}\right)<0$. Let $M=h\left(\sqrt{-\mu_{1} / 3 b_{0}}, b_{0}\right)$ and

$$
\lambda_{*}=-\frac{M \int_{0}^{1} v(x) d x}{\int_{0}^{1} f(x) v(x) d x},
$$

and it follows from (64) that $u(\xi)<0$ for $\lambda>\lambda_{*}$. Therefore, there exists a $\lambda_{*}>0$ such that (1) has no positive solution for $\lambda>\lambda_{*}$.

By applying Lemma 14 , we obtain that $u_{*}(x)$ is still a positive solution of (1) for $\lambda=\lambda_{*}$. We denote the degenerate solution $\left(\lambda_{*}, u_{*}\right)$. At $\left(\lambda_{*}, u_{*}\right)$, we verify that Lemma 16 can be applied here. It follows from (43) that $D_{u} F\left(\lambda_{*}, u_{*}\right)[v]=$ $v^{\prime \prime}+\left[a(x)-3 b(x) u_{*}^{2}\right] v=0$. In fact, 0 is simple and the principal eigenvalue of (5), and the first eigenfunction $v(x)>$ 0 ; therefore, $\operatorname{dim} N\left(D_{u} F\left(\lambda_{*}, u_{*}\right)\right)=\operatorname{codim} R\left(D_{u} F\left(\lambda_{*}, u_{*}\right)\right)=$ 1 and the null space $N\left(D_{u} F\left(\lambda_{*}, u_{*}\right)\right)=\operatorname{span}\{v\}$. Therefore, the Fredholm index of $D_{u} F\left(\lambda_{*}, u_{*}\right)$ is zero. Conditions (1) and (2) of Lemma 16 are satisfied. Next we verify Condition (3) of Lemma 16. Suppose on the contrary that $D_{\lambda} F\left(\lambda_{*}, u_{*}\right)=$ $-f(x) \in R\left(D_{u} F\left(\lambda_{*}, u_{*}\right)\right)$; namely, there is a continuous function $w$ satisfying

$$
w^{\prime \prime}+\left[a(x)-3 b(x) u_{*}^{2}\right] w=-f(x) .
$$

Consider the linearized equation of $(1)$ at $\left(\lambda_{*}, u_{*}\right)$

$$
v^{\prime \prime}+\left[a(x)-3 b(x) u_{*}^{2}\right] v=0 \text {. }
$$

Since $F$ is singular at $\left(\lambda_{*}, u_{*}\right)$, that is, (67) has a nontrivial solution $v(x)$ such that $v(x)>0$. Multiplying (66) by $v$, subtracting from (67) multiplied by $w$ and integrating by parts on $[0,1]$, we have

$$
0=\int_{0}^{1} v^{\prime \prime} w-w^{\prime \prime} v d x=\int_{0}^{1} v(x) f(x) d x,
$$

a contradiction, since both $v(x)$ and $f(x)$ are positive. Therefore, Condition (3) of Lemma 16 is satisfied. Near $\left(\lambda_{*}, u_{*}\right)$, the solutions of

$$
F(\lambda(s), u(s))=0, \quad \text { for all } s \in[-\delta, \delta]
$$

form a curve

$$
\begin{aligned}
& \left\{(\lambda(s), u(s))=\left(\lambda_{*}+\tau(s), u_{*}+s v+z(s)\right) \mid s\right. \\
& \left.\in[-\delta, \delta],(\lambda(0), u(0))=\left(\lambda_{*}, u_{*}\right)\right\} .
\end{aligned}
$$

Differentiating (69) twice in $s$, setting $s=0$ and $\left.u_{s}\right|_{s=0}=v(x)$, and evaluating at $\left(\lambda_{*}, u_{*}\right)$, we have

$$
\begin{aligned}
u_{s s}^{\prime \prime} & +\left[a(x)-3 b(x) u_{*}^{2}\right] u_{s s} \\
& =6 b(x) u_{*} v^{2}+\tau_{s s}(0) f(x) .
\end{aligned}
$$

Multiplying the linearized equation (67) by $u_{s s}$, subtracting from (71) multiplied by $v$, and integrating by parts over $[0,1]$, we obtain

$$
\tau_{s s}(0)=-\frac{6 \int_{0}^{1} b(x) u_{*} v^{3} d x}{\int_{0}^{1} f(x) v(x) d x}<0 .
$$

Therefore, $\left(\lambda_{*}, u_{*}\right)$ is a fold point of $F(\lambda, u)$ to the left. It follows from the above that the curve of positive solutions cannot be continued to the right indefinitely for all $\lambda>0$. Hence, the positive solution curve will make a left turn at $\left(\lambda_{*}, u_{*}\right)$. Near the critical point $\left(\lambda_{*}, u_{*}\right)$, by the CrandallRabinowitz bifurcation theorem, there are two branches of positive solutions denoted by the upper branch $u_{+}(x, \lambda)$ and the lower branch $u_{-}(x, \lambda)$ with $u_{-}(x, \lambda)<u_{+}(x, \lambda)$. It follows from Lemmas 14, 15, and 18 that the fold point $\left(\lambda_{*}, u_{*}\right)$ is unique, and the upper branch $u_{+}(x, \lambda)$ is monotone decreasing for all $\lambda<\lambda_{*}$ and lower branch $u_{-}(x, \lambda)$ is monotone increasing for all $0<\lambda<\lambda_{*}$. Therefore, the lower branch curve is monotone increasing and continues to the left without any turnings. Rewriting (1) in the following form:

$$
u^{\prime \prime}+\left(a(x)-b(x) u^{2}\right) u=\lambda f(x)
$$

since $a(x)-b(x) u^{2}<a(x) \ll \pi^{2} / 4$, it follows from Lemma 14 that $u(x)>0$ or $u(x)<0$. This shows that the solution changes its sign only at $(\lambda, u)=(0,0)$. Since the nonlinearity $g(x, u)=a(x) u-b(x) u^{3}$ is an odd function in $u$, it follows from the symmetry that if $(\lambda, u(x))$ is a solution of $(1)$, so is $(-\lambda,-u(x))$. Thus, the component of solutions we constructed above forms a smooth reversed $S$-shaped curve with exactly two turning points $\left(\lambda_{*}, u_{*}\right)$ and $\left(-\lambda_{*},-u_{*}\right)$.

It follows from Lemma 18 that the positive solution $u_{0}$ is stable. For $\lambda<\lambda^{*}$, the upper branch $u_{+}(x, \lambda)$ remains stable until it reaches the degenerate solution $\left(\lambda_{*}, u_{*}\right)$. Next we prove the lower branch $u_{-}(x, \lambda)$ is unstable. Let $u_{m}$ be any one solution of lower branch $u_{-}(x, \lambda)$ such that $0<$ $u_{m}<u_{*}$. Since $a(x)-3 b(x) u_{m}^{2}>a(x)-3 b(x) u_{*}^{2}$, we have $\mu_{1}\left(a(x)-3 b(x) u_{m}^{2}\right)<\mu_{1}\left(a(x)-3 b(x) u_{*}^{2}\right)=0$. Therefore, the lower branch $u_{-}(x, \lambda)$ is unstable. Since the solution set is symmetric with respect to the origin, the stability of negative solutions $u(x)$ is obtained by using the property of symmetry. The upper branch of the negative solutions is unstable and the lower branch of negative solutions is stable. Therefore, all solutions of (1) lie on a unique reversed S-shaped solution curve. This completes the proof. 


\section{Data Availability}

No data were used to support this study.

\section{Conflicts of Interest}

The authors declare that there are no conflicts of interest regarding the publication of this paper.

\section{Acknowledgments}

This work was completed with the support of Tian Yuan Special Funds of the National Science Foundation of China (no. 11626182).

\section{References}

[1] J. Chu, X. Lin, D. Jiang, D. O’Regan, and R. P. Agarwal, “Positive solutions for second-order superlinear repulsive singular Neumann boundary value problems," Positivity. An International Mathematics Journal Devoted to Theory and Applications of Positivity, vol. 12, no. 3, pp. 555-569, 2008.

[2] Z. Li, "Existence of positive solutions of superlinear secondorder Neumann boundary value problem," Nonlinear Analysis. Theory, Methods \& Applications. An International Multidisciplinary Journal, vol. 72, no. 6, pp. 3216-3221, 2010.

[3] G. Bonanno and A. Sciammetta, "Existence and multiplicity results to Neumann problems for elliptic equations involving the p-Laplacian," Journal of Mathematical Analysis and Applications, vol. 390, no. 1, pp. 59-67, 2012.

[4] E. Sovrano and F. Zanolin, "Indefinite weight nonlinear problems with Neumann boundary conditions," Journal of Mathematical Analysis and Applications, vol. 452, no. 1, pp. 126-147, 2017.

[5] G. Feltrin and E. Sovrano, "Three positive solutions to an indefinite Neumann problem: a shooting method," Nonlinear Analysis. Theory, Methods \& Applications. An International Multidisciplinary Journal, vol. 166, pp. 87-101, 2018.

[6] J. Llibre and L. A. Roberto, "On the periodic solutions of a class of Duffing differential equations," Discrete and Continuous Dynamical Systems - Series A, vol. 33, no. 1, pp. 277-282, 2013.

[7] H. Chen and Y. Li, "Stability and exact multiplicity of periodic solutions of Duffing equations with cubic nonlinearities," Proceedings of the American Mathematical Society, vol. 135, no. 12, pp. 3925-3932, 2007.

[8] A. Lomtatidze and J. Sremr, "On periodic solutions to secondorder Duffing type equations," Nonlinear Analysis: Real World Applications, vol. 40, pp. 215-242, 2018.

[9] C.-C. Tzeng, K.-C. Hung, and S.-H. Wang, "Global bifurcation and exact multiplicity of positive solutions for a positone problem with cubic nonlinearity," Journal of Differential Equations, vol. 252, no. 12, pp. 6250-6274, 2012.

[10] S.-H. Wang and T.-S. Yeh, "A complete classification of bifurcation diagrams of a Dirichlet problem with concave-convex nonlinearities," Journal of Mathematical Analysis and Applications, vol. 291, no. 1, pp. 128-153, 2004.

[11] K.-C. Hung and S.-H. Wang, "A theorem on S-shaped bifurcation curve for a positone problem with convex-concave nonlinearity and its applications to the perturbed Gelfand problem," Journal of Differential Equations, vol. 251, no. 2, pp. 223-237, 2011.
[12] H. Pan and R. Xing, "Time maps and exact multiplicity results for one-dimensional prescribed mean curvature equations," Nonlinear Analysis. Theory, Methods \& Applications. An International Multidisciplinary Journal, vol. 74, no. 4, pp. 1234-1260, 2011.

[13] H. Chen, H. Xing, and X. He, "Bifurcation and stability of solutions to a logistic equation with harvesting," Mathematical Methods in the Applied Sciences, vol. 38, no. 8, pp. 1623-1630, 2015.

[14] P. Cl'ement and G. Sweers, "Uniform anti-maximum principles," Journal of Differential Equations, vol. 164, no. 1, pp. 118-154, 2000.

[15] W. Reichel, "Sharp parameter ranges in the uniform antimaximum principle for second-order ordinary diffrential operators," Zeitschrift fur Angewandte Mathematik und Physik, vol. 54, no. 5, pp. 822-838, 2003.

[16] M. G. Crandall and P. H. Rabinowitz, "Bifurcation, perturbation of simple eigenvalues and linearized stability," Archive for Rational Mechanics and Analysis, vol. 52, pp. 161-180, 1973.

[17] H. Kielhöfer, Bifurcation Theory: An Introduction with Applications to PDEs, Springer, New York, NY, USA, 2003.

[18] S. Oruganti, J. Shi, and R. Shivaji, "Diffusive logistic equation with constant yield harvesting. I. Steady states," Transactions of the American Mathematical Society, vol. 354, no. 9, pp. 36013619, 2002.

[19] K. Deimling, Nonlinear Functional Analysis, Springer, Berlin , Germany, 1985.

[20] J. X. Sun, Nonlinear Functional Analysis and Its Applications, Science Press, Beijing, China, 2007.

[21] H. Xing, H. Chen, and X. He, "Exact multiplicity and stability of solutions of second-order Neumann boundary value problem," Applied Mathematics and Computation, vol. 232, pp. 1104-1111, 2014. 


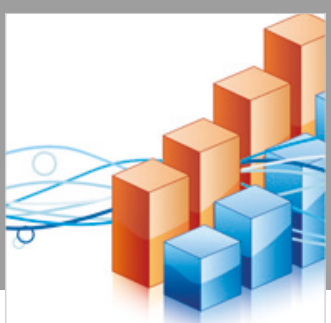

Advances in

Operations Research

\section{-n-m}
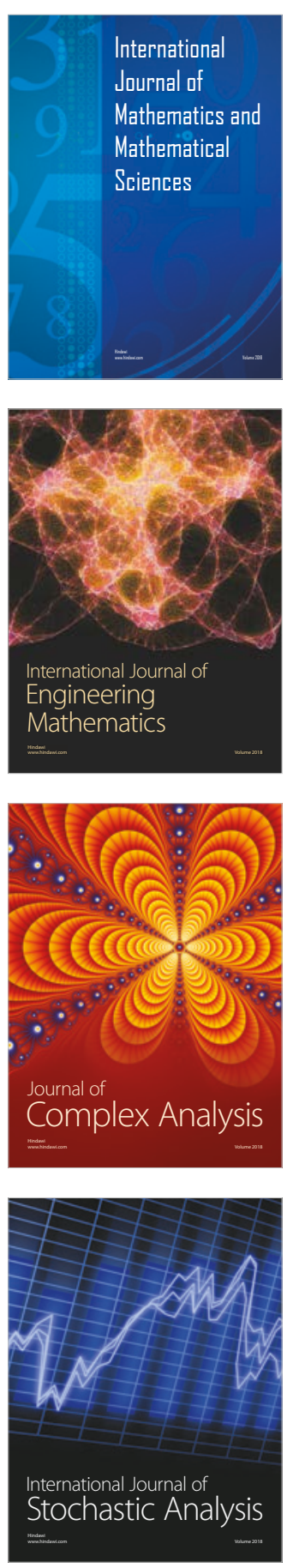
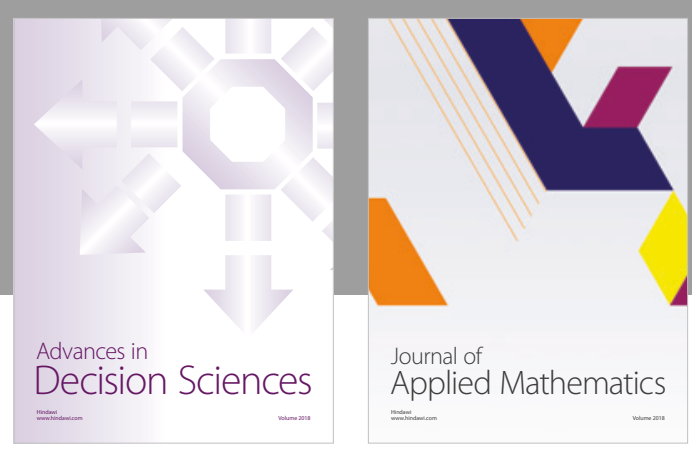

Journal of

Applied Mathematics
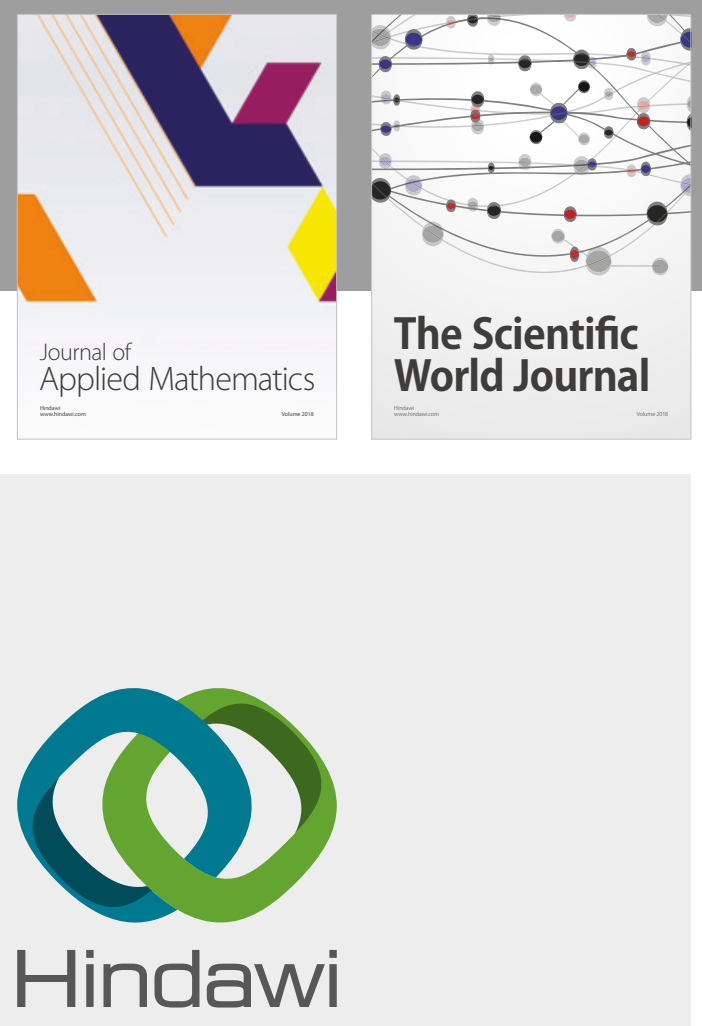

Submit your manuscripts at

www.hindawi.com

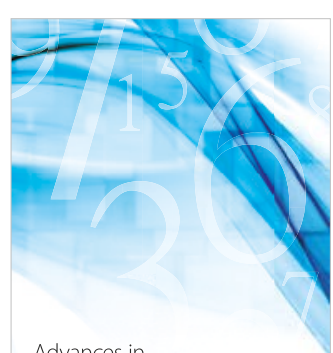

Advances in
Numerical Analysis
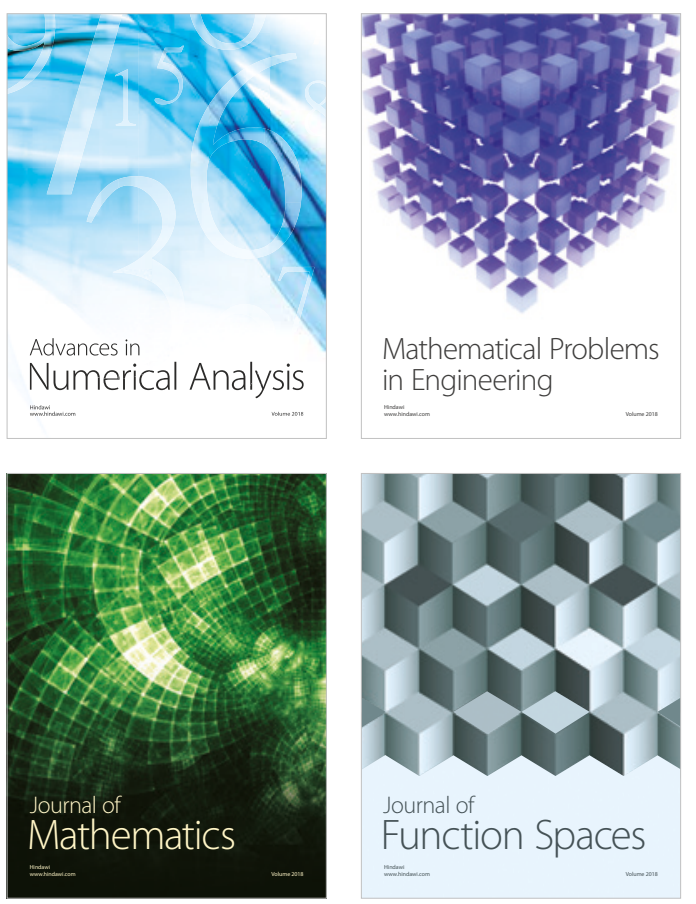

Mathematical Problems in Engineering

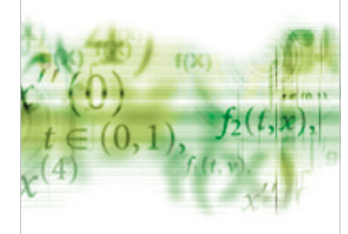

International Journal of

Differential Equations

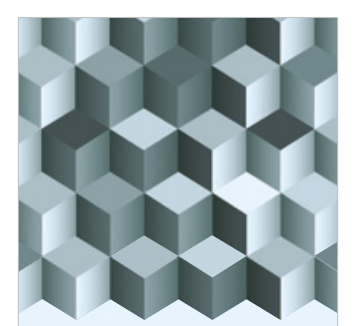

Journal of

Function Spaces

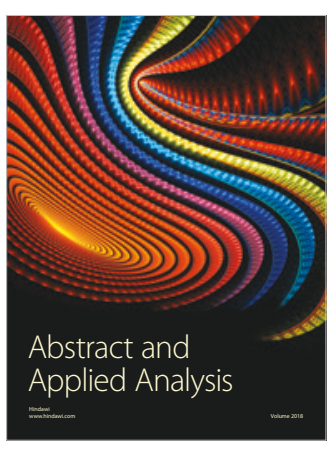

The Scientific

World Journal

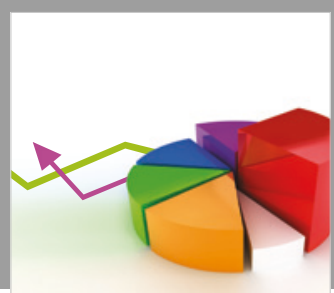

Journal of

Probability and Statistics
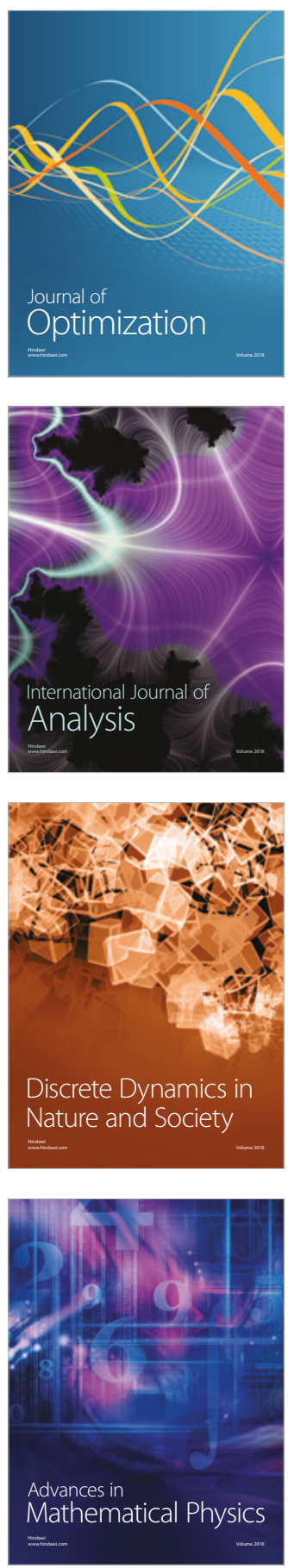